\title{
Oxazepam and cognitive reappraisal: a randomised experiment
}

Gustav Nilsonne ${ }^{1,2^{*}}$, Sandra Tamm ${ }^{1,2,3}$, Armita Golkar ${ }^{1,4}$, Andreas Olsson ${ }^{1}$, Karolina Sörman ${ }^{1}$, Katarina Howner ${ }^{1}$, Marianne Kristiansson ${ }^{1}$, Martin Ingvar ${ }^{1}$, Predrag Petrovic $^{1}$

1 Karolinska Institutet, Department of Clinical Neuroscience, Nobels väg 9, SE-171 77 Stockholm, Sweden

2 Stockholm University, Department of Psychology

3 University of Oxford, Department of Psychiatry

4 University of Amsterdam, Department of Clinical Psychology

* gustav.nilsonne@ki.se

\section{Abstract}

Background: Cognitive reappraisal is a strategy for emotional regulation, important in the context of anxiety disorders. It is not known whether anxiolytic effects of benzodiazepines affect cognitive reappraisal.

Aims: We aimed to investigate the effect of $25 \mathrm{mg}$ oxazepam on cognitive reappraisal. Methods: In a preliminary investigation, 33 healthy male volunteers were randomised to oxazepam or placebo, and then underwent an experiment where they were asked to use cognitive reappraisal to upregulate or downregulate their emotional response to images with negative or neutral emotional valence. We recorded unpleasantness ratings, skin conductance, superciliary corrugator muscle activity, and heart rate. Participants completed rating scales measuring empathy (Interpersonal Reactivity Index, IRI), anxiety (State-Trait Anxiety Inventory, STAI), alexithymia (Toronto Alexithymia Scale-20, TAS-20), and psychopathy (Psychopathy Personality Inventory-Revised, PPI-R).

Results: Upregulation to negative-valence images in the cognitive reappraisal task caused increased unpleasantness ratings, corrugator activity, and heart rate compared to downregulation. Upregulation to both negative- and neutral-valence images caused increased skin conductance responses. Oxazepam caused lower unpleasantness ratings to negative-valence stimuli, but did not interact with reappraisal instruction on any outcome. Self-rated trait empathy was associated with stronger responses to negative-valence stimuli, whereas self-rated psychopathic traits were associated with weaker responses to negative-valence stimuli.

Conclusions: While $25 \mathrm{mg}$ oxazepam caused lower unpleasantness ratings in response to negative-valence images, we did not observe an effect of $25 \mathrm{mg}$ oxazepam on cognitive reappraisal.

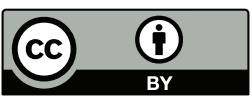




\section{Introduction}

\section{Background}

Emotional regulation is an important aspect of normal behavior in healthy individuals and often altered in patients with psychiatric disorders, including emotional instability and anxiety [1.2], suggesting a less effective top-down control of emotional processes. Pharmacological substances such as benzodiazepines provide a rapid anxiolytic effect, but are associated with a risk for dependency. Anecdotally, benzodiazepines have been reported to be used to disinhibit criminal violent behavior 3 [5]. This disinhibition theory of criminal violent behaviour suggests that either empathy processes or top-down regulatory processes are suppressed by benzodiazepines. However, previous studies have not tested whether benzodiazepines affect top-down regulation of emotion.

One strategy to regulate emotions is through reappraising emotional stimuli in a non-emotional way. Cognitive reappraisal represents an explicit top-down regulatory mechanism in the processing of emotional stimuli $[6$. In functional brain imaging studies, cognitive reappraisal has been associated with activity particularly in dorsolateral prefrontal cortex (dlPFC) and lateral orbitofrontal cortex (OFC) 7 -11. These areas are proposed to exert top-down control over emotional processing in limbic brain structures including the amygdala $7-\sqrt{12}$. Cognitive reappraisal is important in psychiatric conditions involving anxiety [13 15], which may be associated with insufficient top-down control [14,16. One meta-analysis of fMRI studies found that patients with mood and anxiety disorders showed less activation than healthy controls during cognitive reappraisal in areas including the bilateral dorsomedial prefrontal cortex and the left ventrolateral prefrontal cortex 17. Training in cognitive reappraisal can also be part of a treatment for depression, as well as other psychiatric conditions (reviewed in [18])

Benzodiazepines are anxiolytic drugs acting through $\mathrm{GABA}_{\mathrm{A}}$ receptors, which are pentameric ligand-gated ion channels composed of $\alpha, \beta$, and $\gamma$ subunits. Anxiolytic effects of benzodiazepines are suggested to be mediated primarily by the $\alpha-2$ subunit containing $\mathrm{GABA}_{\mathrm{A}}$ receptors [19, expressed particularly in the amygdala [20]. Conversely, sedative and anticonvulsant effects are likely mediated mainly by $\alpha-1$ subunit containing $\mathrm{GABA}_{\mathrm{A}}$ receptors, highly expressed in the cerebral cortex 19$] 22$. In laboratory settings, benzodiazepines have been shown to enhance the response to positive vs negative words, modulate emotional memory, and inhibit recognition of facial expressions of anger 23,24. It has been shown that increased activity in amygdala and insula associated to fear and anxiety processing are suppressed in a dose-dependent manner by treatment with benzodiazepines in humans $12,25,26$. One benzodiazepine with a clear anxiolytic effect, but with less sedative properties than many other benzodiazepines is oxazepam. We have previously tested the hypothesis that $20 \mathrm{mg}$ oxazepam would inhibit empathy for pain, finding no conclusive evidence for such an effect 27]. Another possible explanation for instrumental use of benzodiazepines could be that they, often in combination with alcohol, reduce the ability to regulate emotional responses and thereby cause increased aggression. This would suggest an interaction between top-down regulatory mechanisms and treatment with benzodiazepines. This hypothesis is especially interesting since areas in prefrontal cortex that are involved in emotional regulation have high concentrations of $\mathrm{GABA}_{\mathrm{A}}$ receptors, suggesting a putative mechanism by which the $\mathrm{GABA}_{\mathrm{A}}$ system may impact emotional regulation efficiency 28 .

Thus, both cognitive reappraisal and benzodiazepines may be associated with inhibition of unpleasantness in response to negatively valenced emotional stimuli, and both act upon the amygdala. However, it is not known whether these regulatory processes interact or act independently on emotional processing. 
We investigated the effects of $25 \mathrm{mg}$ oxazepam in a study encompassing three different experiments targeting different types of emotional processing: emotional mimicry, empathy for pain, and cognitive reappraisal. This paper reports results from the cognitive reappraisal experiment. Results from the experiments on emotional mimicry and empathy for pain have been previously reported [27], and the primary findings were that $25 \mathrm{mg}$ oxazepam did not have a major effect on emotional mimicry nor on empathy for pain. Cognitive reappraisal can be thought of as a higher-level top-down emotion regulating function, mechanistically distinct from the more bottom-up processes of emotional mimicry and empathy for pain.

\section{Aims}

We aimed to investigate effects of $25 \mathrm{mg}$ oxazepam and cognitive reappraisal on emotion-related outcomes. The main hypothesis was that oxazepam would be associated with a reduced ability to regulate emotions through cognitive reappraisal. To capture subjective experience as well as psycho-physiological aspects of emotion, we investigated participants' ratings of unpleasantness, skin conductance, heart rate and facial EMG. Additionally, we explored associations of personality traits related to empathy, psychopathy and anxiety to emotional regulation.

\section{Materials and Methods}

\section{Study design}

This experiment formed part of a larger study on the effects of oxazepam on social emotional processes. For a detailed description, see [27. Briefly, participants were randomised to $25 \mathrm{mg}$ oxazepam or placebo in a double-blind between-groups design, and underwent experiments on emotional mimicry, empathy for pain, and cognitive reappraisal. This paper reports effects of oxazepam on cognitive reappraisal. The study was performed in two waves. Stimuli were balanced over regulation instructions using two different trial lists. Due to an error in randomisation in wave 1, stimulus images were not balanced between instructions to upregulate and downregulate emotional response. Therefore only data from wave 2 were analysed for the experiment on cognitive reappraisal.

\section{Participants}

As described in 27, participants were required to be right-handed, male, 18-45 years of age, to have no history of neurological or psychiatric disease including substance abuse, to speak and understand Swedish fluently, and not to be habitual consumers of nicotine, to reduce the risk of abstinence symptoms during the experiment. Furthermore, students of psychology, behavioural sciences, and medicine (past the 3rd semester) were not included, because training in medicine may cause a more detached attitude towards images of injured and sick people, which were used in the experiment, and because students of psychology and behavioural science may be prone to metacogitate and use different strategies for emotional regulation. We aimed for a sample size of $n=40$, with 20 participants in each of the two treatment groups, based on pragmatic considerations. Participants were paid 500 SEK (approx. 50 Euro or 60 USD), subject to tax.

\section{Stimuli and experimental paradigm}

The experimental paradigm was adapted from 7 . Participants were shown negative and neutral stimuli following an instruction to either upregulate or downregulate their 


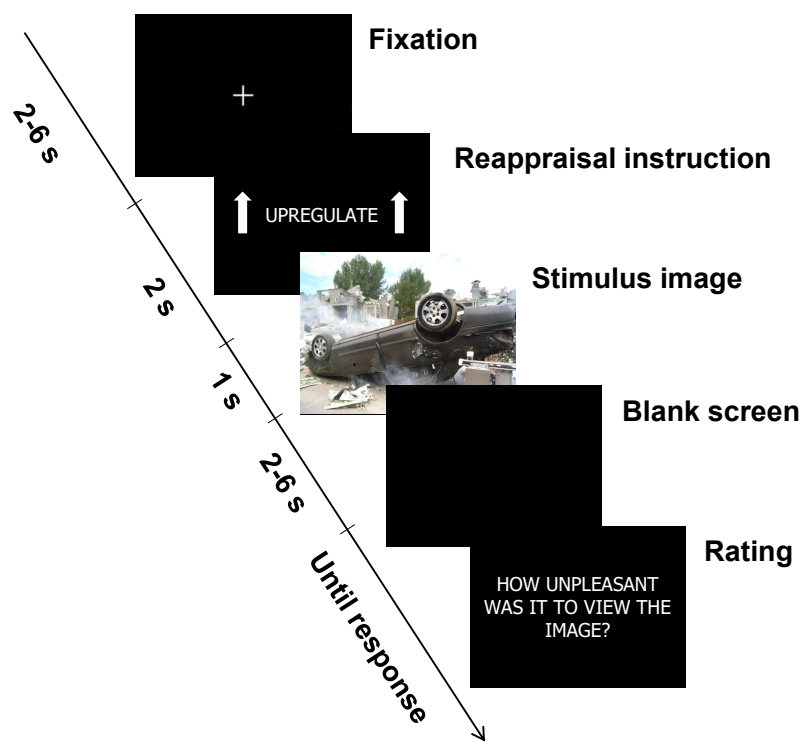

64 events; 16 of each category: downregulate/upregulate, neutral/negative

Fig 1. Experimental paradigm. The example stimulus image shown here was not part of the stimulus set.

emotional response, see Fig 1. The reappraisal instruction was shown for 2 seconds, followed immediately by the image, which was shown for 1 second. Stimulus images were chosen from the International Affective Picture System (IAPS) 29. On normative ratings, negative images had a mean valence of 20.2 [SD 0.25], and neutral images had a mean valence of 5.02 [0.05]. Participants were asked to either upregulate or downregulate their emotional response by cognitive reappraisal, i.e. imagining different contexts for the situations shown in the images, such as a fictitious situation (downregulation) or something happening to someone close to them (upregulation). Participants were specifically instructed not to close their eyes or look away. In total, each participant was shown 64 trials, 16 of each category (upregulate/downregulate, neutral/negative). After each stimulus, participants were asked to rate their perceived unpleasantness on a visual analog scale from 0 to 100. Before the experiment, participants underwent a demonstration session, and were then asked to explain the instructions back to the experimenter in order to ensure that the instructions were understood. Stimuli were shown using the Presentation software (Neurobehavioral systems, Inc., Berkeley, CA, USA) on a computer screen. Stimulus presentation code is available at https://doi.org/10.5281/zenodo.31480. For a further verification that emotional regulation took place, we administered a recall test online, where participants were asked to say whether they recalled images from the experiment, as well as valence-matched images from the IAPS that had not been shown in the experiment. The data from the recall test were unfortunately lost when the online test platform upgraded their software.

\section{Physiological measures}

We recorded skin conductance, electromyographic (EMG) activity over the superciliary corrugator muscle, and heart rate, as described in 27. 
Briefly, skin conductance responses were measured using $\mathrm{Ag} / \mathrm{AgCl}$ finger electrodes (TSD203, Biopac Systems, Inc.), connected to a GSR100C amplifier (Biopac Systems, Inc.) with the following acquisitions settings: $5 \mu \mho / \mathrm{V}, 1 \mathrm{~Hz}$ low-pass filter, and direct current. To remove non-physiological noise, data were further filtered in the Acqknowledge software using a low pass filter with a $1 \mathrm{~Hz}$ cutoff and 4000 coefficients and converted from direct to alternating current using an $0.05 \mathrm{~Hz}$ high pass filter. Responses were averaged over a time window of 2 seconds. The time window for analysis was chosen based on inspection of data.

EMG was measured following established guidelines [30], with $4 \mathrm{~mm} \mathrm{Ag}-\mathrm{AgCl}$ electrodes (EL254S, Biopac Systems, Inc.) connected to EMG100C amplifiers (Biopac Systems, Inc.) with the following acquisition settings: gain 500, low-pass filter $500 \mathrm{~Hz}$, notch filter off, and high-pass filter $10 \mathrm{~Hz}$. Sampling was at $1000 \mathrm{~Hz}$. The signal was further filtered in the Acqknowledge software using a band pass filter of 30 to $300 \mathrm{~Hz}$ to remove signal not due to muscle activity. A band stop filter at 49 to $51 \mathrm{~Hz}$ was used to filter out line noise. Average rectified EMG signal was determined. Recordings were downsampled to $100 \mathrm{~Hz}$ in order to decrease file size, and data were exported as text files. Before analyses, recordings were further downsampled to $10 \mathrm{~Hz}$ using a loess curve in R. Responses were averaged over a time window of 2 seconds and log-transformed before statistical analysis, in order to better approximate a normal distribution. The time window for analysis was chosen based on inspection of data.

A 3-lead EKG was acquired using Ag/ $\mathrm{AgCl}$ electrodes (EL503, Biopac Systems, Inc.) with ECG100 amplifiers (Biopac Systems, Inc.) with the following settings: gain 2000, mode $\mathrm{R}$ wave, $35 \mathrm{HzLPN}$ on, high-pass filter $0.5 \mathrm{~Hz}$. Sampling was at $1000 \mathrm{~Hz}$.

Recordings were downsampled to $100 \mathrm{~Hz}$ in order to decrease file size, and data were exported from the Acqknowledge software as text files. Heart rate was derived from raw curves by a peak finding algorithm in R. Estimated heart rate of $<40$ or $>200$ beats per minutes was rejected $(0.2 \%$ of data). For each event, heart rate was normalised to the 2 seconds preceding regulation instruction onset and averaged over a time window from 3 to 5 seconds from regulation instruction onset. The time window for analysis was chosen based on inspection of data.

\section{Rating scales}

The interpersonal reactivity index (IRI) has four subscales which measure different dimensions of trait empathy: empathic concern (EC), perspective taking (PT), personal distress (PD) and fantasy (FS) [31,32]. The IRI has been validated in a Swedish context [33, although the four-factor structure could not be replicated. Instead, EC formed one factor and PT, PD and FS together formed another factor. For this reason, we have not analysed differences between IRI subscales.

The state-trait anxiety inventory (STAI) has a state and a trait subscale 34 . We used a non-validated Swedish translation with which we have considerable experience, and which can be found in [35]. The state subscale (S) was administered before the experiment, and then again at the end of the experiment.

The Toronto Alexithymia Scale-20 (TAS-20) measures alexithymia, a construct thought to represent difficulties in identifying and describing one's own emotions. It has three subscales: difficulty identifying feelings, difficulty describing feelings and externally oriented thinking [36]. We analysed only total scores. The scale has been validated in Swedish 37.

The psychopathy personality inventory-revised (PPI-R) assesses psychopathic traits 38, 39]. It contains eight content scales, which have been organized into a two-factor structure, encompassing the factors fearless dominance (FD; reflecting social poise, fearlessness and stress immunity) and self-centred impulsivity (SCI; reflecting impulsivity, irresponsibility and egocentricity). It also contains a subscale particularly 
reflecting lack of empathy (coldheartedness, C), which typically does not load highly on either factor. The Swedish version of the PPI-R has been validated based partly on the data collected in this study 40 . For more details on the used rating scales, please see 27 .

\section{Analyses and data}

Data and analysis code for this paper are openly available at https://doi.org/10.5281/zenodo.3903120. In order to preserve anonymity, participants' age and educational background have been omitted from the published dataset. All analyses were made with R 41, using the packages RCurl 42 to read data from GitHub, quantmod [43 to find EKG R wave peaks, nlme 44] to build mixed-effects models, effects 45 to get confidence intervals on estimates, and RColorBrewer 46] for graphing. Mixed-effects models have been used throughout unless otherwise indicated. Effects were deviation coded, meaning that reported effect sizes refer to the difference from the grand mean. For instance, the effect of upregulation refers to whether participants were instructed to upregulate or downregulate, and the reported effect size is the difference between the upregulate condition and the mean of the upregulate and downregulate conditions.

To investigate interaction effects of self-rated personality traits with stimulus valence and reappraisal instruction, a separate regression model was run with each rating scale, in which interactions between the scale score and valence and reappraisal instruction, respectively, were specified. Scale scores were $z$-transformed to yield standardized regression coefficients.

A threshold of $\alpha<0.05$ for statistical significance was used because this threshold is conventional in the field.

\section{Results}

\section{Participants}

Thirty-nine participants were randomised. Six did not perform the reappraisal experiment since they reported having previously participated in other experiments involving viewing images likely to be from the same IAPS stimulus set. The final sample included 33 participants. Participant characteristics are shown in table 1.

Table 1. Participant characteristics.

\begin{tabular}{|c|c|c|}
\hline & placebo & oxazepam \\
\hline $\mathrm{n}$ & 13 & 20 \\
\hline age, median (range) & $22(18-44)$ & $22.5,18-41$ \\
\hline Interpersonal Reactivity Index — Empathic Concern & $3.77(0.59)$ & $3.79(0.31)$ \\
\hline Interpersonal Reactivity Index — Perspective Taking & $3.51(0.37)$ & $3.41(0.47)$ \\
\hline Interpersonal Reactivity Index — Personal Distress & $2.54(0.39)$ & $2.39(0.53)$ \\
\hline Interpersonal Reactivity Index — Fantasy & $3.37(0.5)$ & $3.23(0.67)$ \\
\hline State-Trait Anxiety Index — Trait & $40.33(5.69)$ & $34.9(6.21)$ \\
\hline Toronto Alexithymia Scale-20 & $41.25(10.32)$ & $37.5(8.02)$ \\
\hline Psychopathy Personality Inventory-Revised — Self-Centred Impulsivity & $160.75(20.1)$ & $140.3(24.74)$ \\
\hline Psychopathy Personality Inventory-Revised — Fearless Dominance & $125.5(11.2)$ & $129.45(15.25)$ \\
\hline Psychopathy Personality Inventory-Revised - Coldheartedness & $35.83(3.07)$ & $36.05(4.89)$ \\
\hline
\end{tabular}

Means and standard deviations are given, unless otherwise indicated. 
Unpleasantness ratings, Neutral-valence stimuli

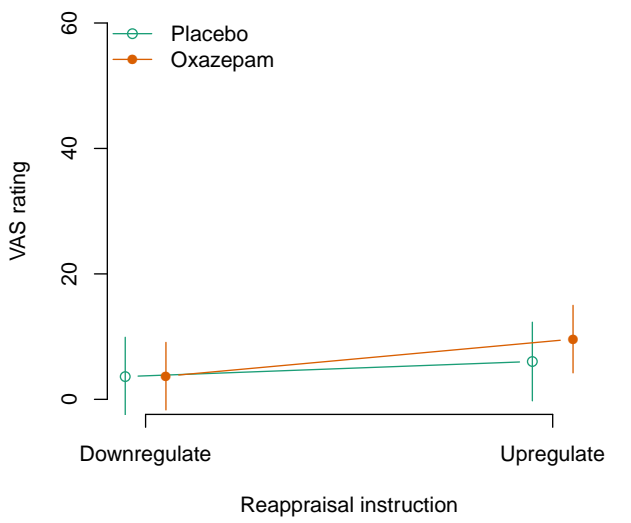

Unpleasantness ratings, Negative-valence stimuli

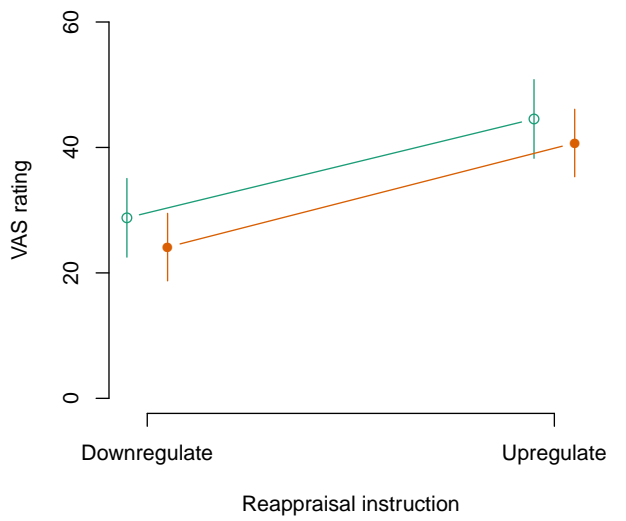

Fig 2. Rated unpleasantness: model estimates with $95 \%$ confidence intervals.

\section{Unpleasantness ratings}

We investigated the interactions between upregulation, negative stimulus valence, and oxazepam treatment on unpleasantness ratings. The three-way interaction was not statistically significant: -2.7 [95\% CI -9.1, 3.8], $p=0.42$ (Fig 2). The two-way interaction between upregulation and negative stimulus valence was statistically significant in the expected direction $(12.0[8.8,15.2], p<0.0001)$. The two-way interaction between negative stimulus valence and oxazepam treatment was statistically significant and showed that lower unpleasantness was reported to negative-valence stimuli in the oxazepam group compared to the placebo group (-6.1 [-9.3, -2.7], $p=0.0002)$, consistent with an anxiolytic effect. The two-way interaction between upregulation and oxazepam treatment was not statistically significant: $2.2[-1.0,5.4]$, $p=0.19$. The main effect of negative stimulus valence was statistically significant: 28.8 $[27.2,30.4], p<0.0001$. The main effect of upregulation was statistically significant: $10.2[8.6,11.8], p<0.0001$, and the main effect of oxazepam treatment was not statistically significant: $-1.2[-9.3,6.9], p=0.76$.

\section{Skin conductance}

Skin conductance was measured as an indicator of autonomic activity. Fig 3 shows time-courses of skin conductance. The time window for signal extraction was chosen based on inspection of time courses.

The three-way interaction between negative stimulus valence, upregulation, and oxazepam treatment was not statistically significant: $0.002[-0.015,0.019], p=0.79$ (Fig 4). The two-way interaction between negative stimulus valence and upregulation was not statistically significant: $-0.006[-0.014,0.003], p=0.18$. The two-way interaction between negative stimulus valence and oxazepam treatment was not statistically significant: $0.002[-0.006,0.011], p=0.57$. The two-way interaction between upregulation and oxazepam treatment was not statistically significant: 0.001 [-0.011, $0.013], p=0.84$. The main effect of negative stimulus valence was not statistically significant: $0.001[-0.004,0.005], p=0.78$. The main effect of upregulation was 0.007 $[0.003,0.011], p=0.001$, as expected. The main effect of oxazepam was not statistically significant: $-0.001[-0.007,0.005], p=0.71$. 
Skin conductance, Neutral-valence stimuli

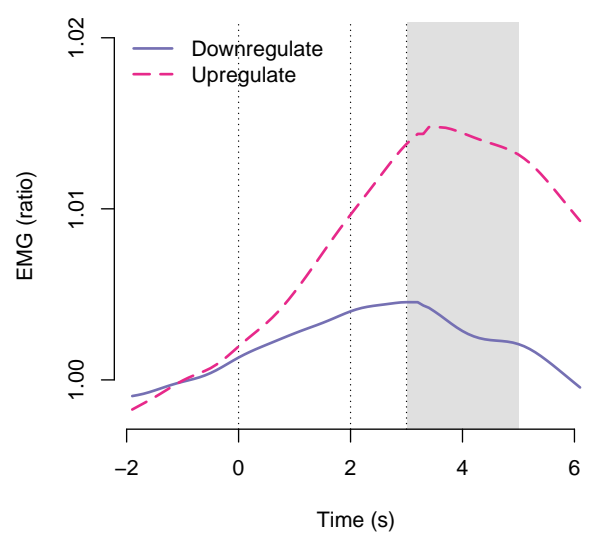

Skin conductance, Negative-valence stimuli

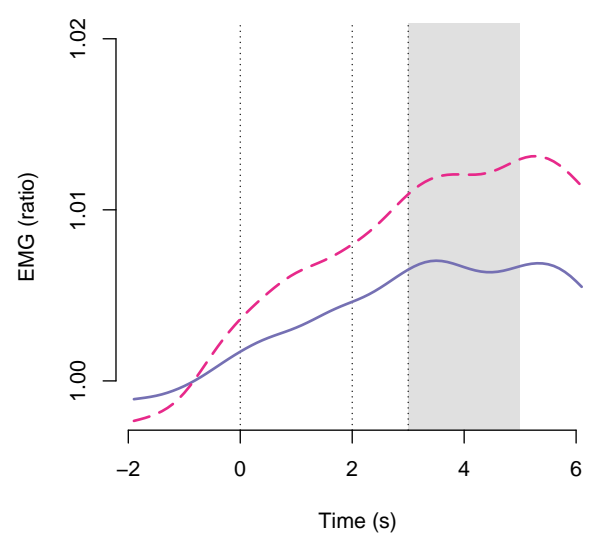

Fig 3. Skin conductance time-courses across treatment groups. The first vertical line shows regulation instruction onset; the second vertical line shows stimulus image onset; and the third vertical line shows stimulus image offset. The shaded gray area shows the time window from which responses were averaged for statistical modelling.

Skin conductance, Neutral-valence stimuli

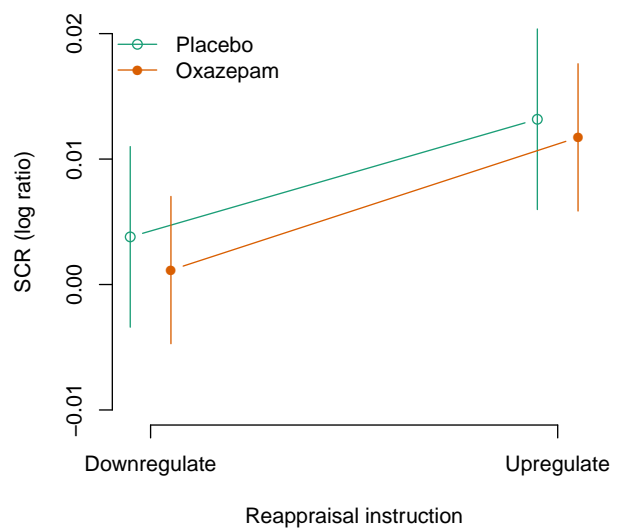

Skin conductance, Negative-valence stimuli

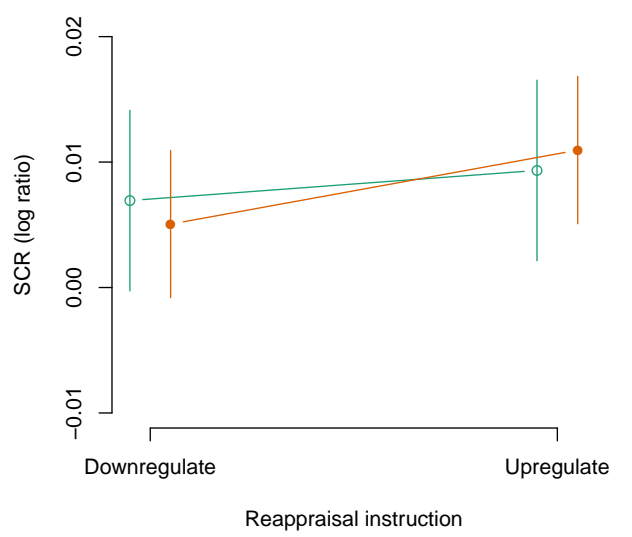

Fig 4. Skin conductance: model estimates with $95 \%$ confidence intervals. 
Corrugator EMG, Neutral-valence stimuli

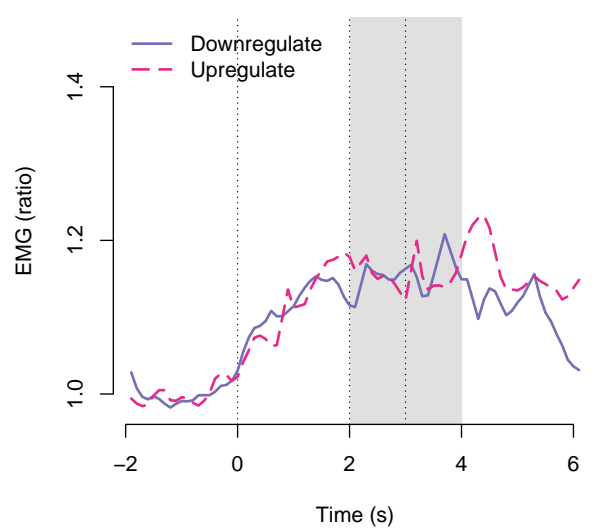

Corrugator EMG, Negative-valence stimuli

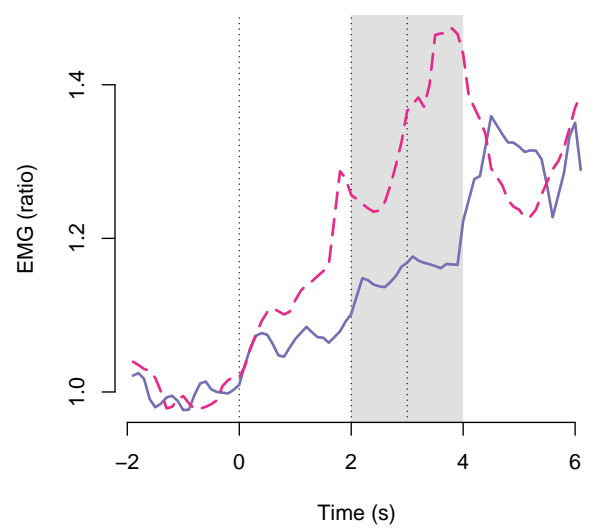

Fig 5. Corrugator EMG time-courses across treatment groups. The first vertical line shows onset of the instruction; the second vertical line shows stimulus image onset; and the third vertical line shows stimulus image offset. The shaded gray area shows the time window from which responses were averaged for statistical modelling.

\section{Corrugator activity}

Activity of the superciliary corrugator muscle was measured as an indicator of negative emotion. Fig 5 shows time-courses of corrugator EMG activity. The time window for signal extraction was chosen based on inspection of time courses.

The three-way interaction between negative stimulus valence, upregulation, and oxazepam treatment was not statistically significant: $-0.031[-0.201,0.138], p=0.72$ (Fig 6). The two-way interaction between negative valence and upregulation was statistically significant: $0.108[0.023,0.192], p=0.01$, as expected, and consistent with unpleasantness ratings. The two-way interaction between negative stimulus valence and oxazepam treatment was not statistically significant: $-0.055[-0.140,0.030], p=0.20$. The two-way interaction between upregulation and oxazepam treatment was not statistically significant: $0.026[-0.059,0.110], p=0.55$. The main effect of negative stimulus valence was statistically significant: $0.061[0.019,0.103], p=0.005$. The main effect of upregulation was statistically significant: $0.069[0.026,0.111], p=0.002$. The main effect of oxazepam treatment was not statistically significant: -0.066 [-0.168, $0.037], p=0.20$.

\section{Heart rate}

Heart rate was measured as an indicator of autonomic activity. Fig 7 shows time-courses of heart rate changes, demonstrating deceleration following stimulus presentation. The time window for signal extraction was chosen based on inspection of time courses.

The three-way interaction between negative valence, upregulation, and oxazepam treatment was not statistically significant: $-0.013[-0.037,0.011], p=0.29$, Fig 8 . The two-way interaction between negative valence and upregulation was statistically significant: $0.012[0.000,0.024], p=0.04$, as expected. The two-way interaction between negative valence and oxazepam treatment was not statistically significant: -0.001 [-0.013, $0.011], p=0.90$. The two-way interaction between upregulation and oxazepam treatment was not statistically significant: $-0.001[-0.013,0.010], p=0.82$. The main effect of negative valence was not statistically significant: $-0.002[-0.008,0.004], p=0.51$. The main effect of upregulation was not statistically significant: 0.003 [-0.003, 0.009], 
Corrugator EMG, Neutral-valence stimuli

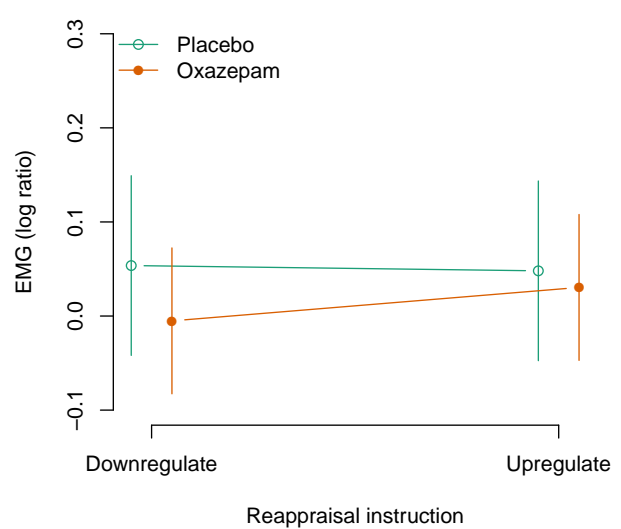

Corrugator EMG, Negative-valence stimuli

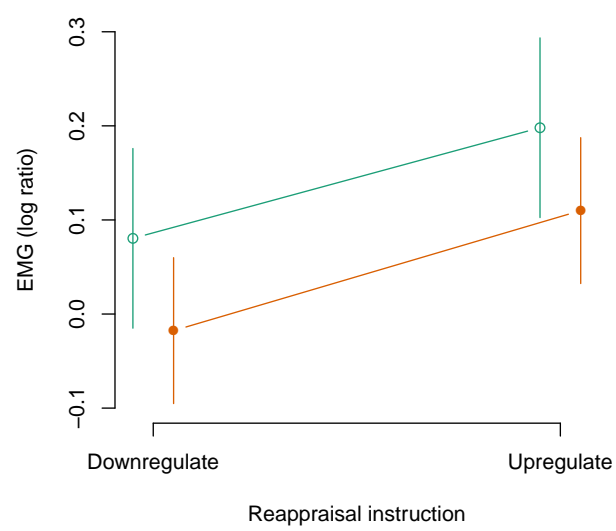

Fig 6. Corrugator EMG responses: model estimates with $95 \%$ confidence intervals.

Heart rate, Neutral-valence stimuli

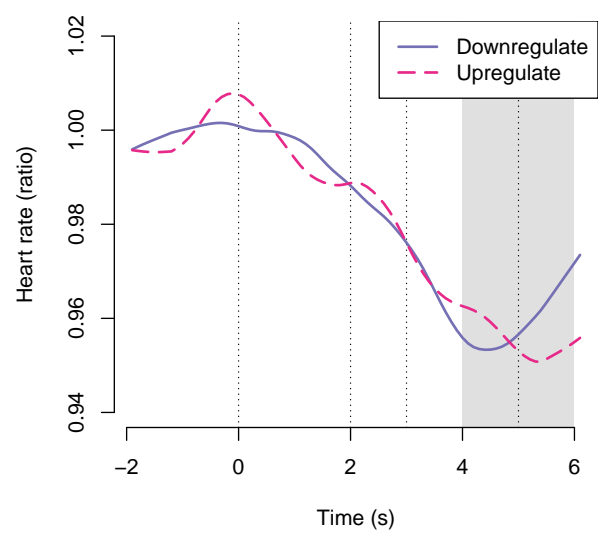

Heart rate, Negative-valence stimuli

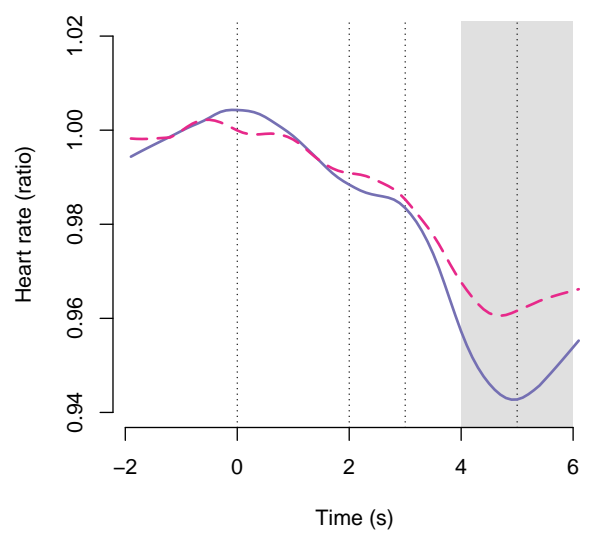

Fig 7. Heart rate time-courses across treatment groups. The first vertical line shows onset of the instruction; the second vertical line shows stimulus image onset; and the third vertical line shows stimulus image offset. The shaded gray area shows the time window from which responses were averaged for statistical modelling. 

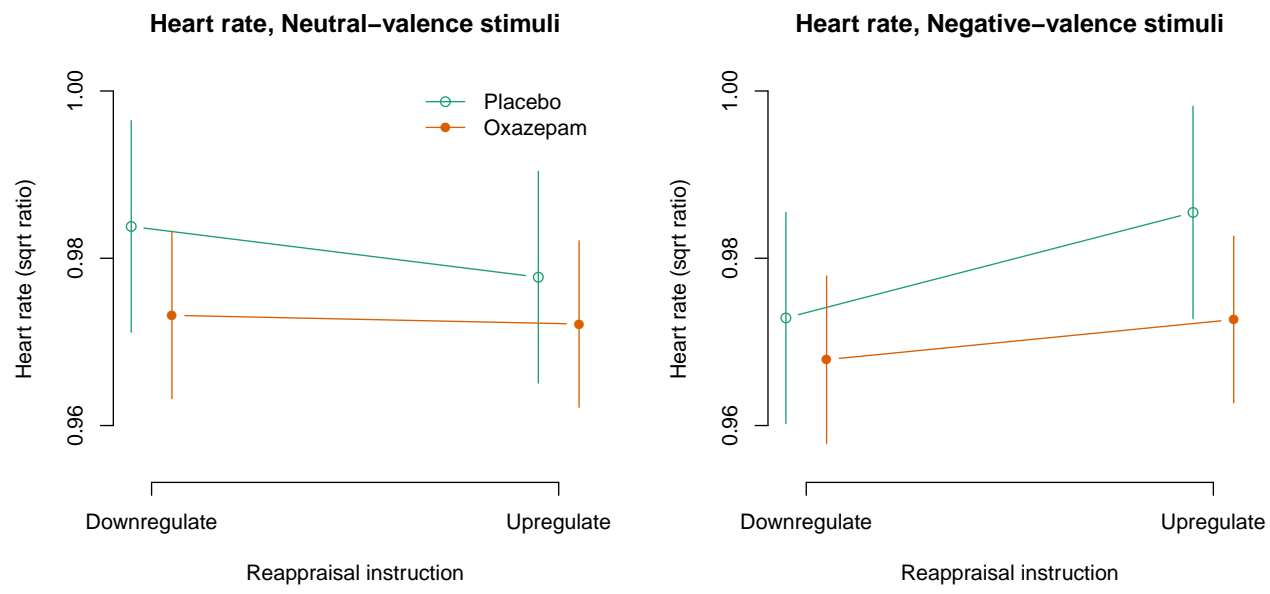

Fig 8. Heart rate: model estimates with $95 \%$ confidence intervals.

$p=0.39$. The main effect of oxazepam treatment was not statistically significant: -0.009 $[-0.021,0.004], p=0.19$, Fig 8

\section{Associations between self-rated personality traits to responses to stimuli and instructions to perform reappraisal}

We performed exploratory analyses of associations between self-rated personality traits and rated unpleasantness of negative vs neutral images (stimulus valence) as well as instruction to upregulate vs downregulate (instruction). The corresponding statistics can be found in (Fig 9). For stimulus valence, we found that empathy subscales IRI-EC, IRI-PT, and IRI-F were associated with higher rated unpleasantness to negative-valence images, as expected. Conversely, empathy subscale IRI-PD was associated to lower rated unpleasantness, contrary to our expectations. All three subscales of the psychopathy personality inventory-revised (PPI-R) were associated with lower rated unpleasantness to negative-valence images, as expected. For physiological measures, the only notable associations between personality traits and stimulus valence was that PPI-R-C (coldheartedness) was associated with higher corrugator activity and lower heart rate in response to negative images.

For instruction, we found that empathy subscales IRI-PD and IRI-F were associated with lower rated unpleasantness when instructed to upregulate, compared to downregulate. For physiological measures, notable associations between regulation instruction and outcome were seen only for corrugator EMG, where the perspective taking empathy subscale IRI-PT and the Fearless Dominance subscale of the PPI-R (PPI-R-FD) were associated with higher activity in the upregulate condition, whereas the Toronto Alexithymia Scale-20 (TAS-20) and the personal distress empathy subscale IRI-PD were associated with lower activity in the upregulate condition.

\section{Discussion}

In this study, we examined the effect of $25 \mathrm{mg}$ oxazepam on cognitive reappraisal. We found that upregulation of negative-valence images caused increased unpleasantness ratings, corrugator activity, and heart rate compared to downregulation, confirming the validity of the paradigm. Oxazepam caused lower ratings of unpleasantness to negative stimuli, but no interaction between oxazepam and reappraisal was observed. 
bioRxiv preprint doi: https://doi.org/10.1101/656355; this version posted February 5, 2021. The copyright holder for this preprint (which was not certified by peer review) is the author/funder, who has granted bioRxiv a license to display the preprint in perpetuity. It is made available under aCC-BY 4.0 International license.
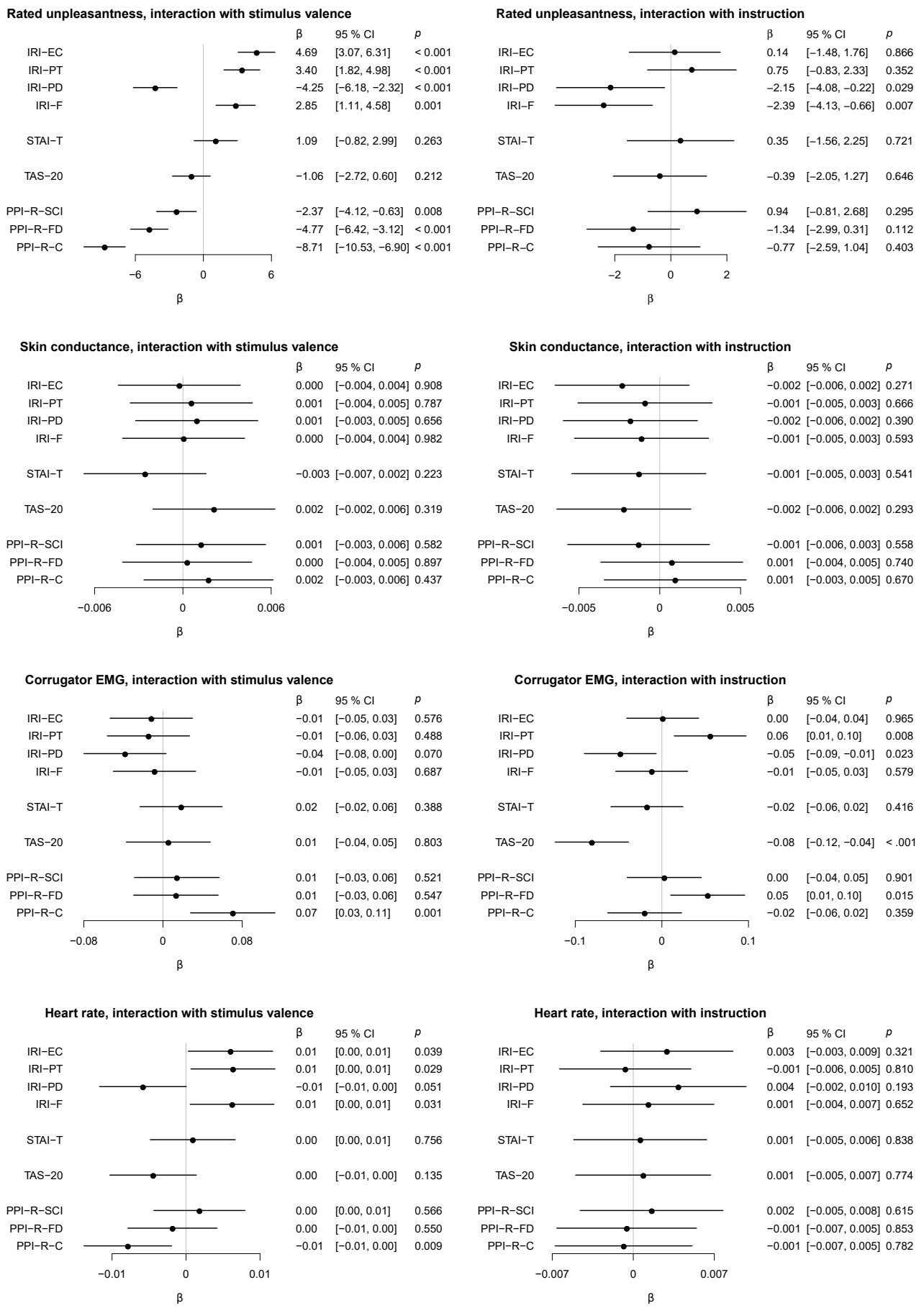

Fig 9. Interaction effects of self-rated personality traits with stimulus valence and reappraisal instruction. Effects shown are standardized regression coefficients with $95 \%$ confidence intervals. 
As we have previously described in a report on the empathy for pain paradigm using the full sample of this experiment, 27, efficacy of drug effects was verified by longer response times and lower rated anxiety after the experiment in the oxazepam group. As noted above, upregulation of negative-valence images caused increased unpleasantness ratings, corrugator activity, and heart rate. Upregulation to both negative- and neutral-valence images also caused increased skin conductance responses. These findings confirm that the experimental paradigm was effective and that the drug reached a biological effect in the oxazepam group, providing an appropriate setting to test the effect of oxazepam on cognitive reappraisal. Ratings of unpleasantness may be affected by demand effects. As argued by Ray et al. [47, it is more convincing to measure both self-report and autonomic indices of emotional responses in reappraisal experiments, since they are sensitive to partly different sets of biases. In particular, autonomic responses are likely to be less affected by demand characteristics. In line with this reasoning, our finding that upregulation by cognitive reappraisal was associated with increased skin conductance responses further supports the validity of the paradigm, and is consistent with earlier findings 10 .

Oxazepam caused lower ratings of unpleasantness to negative stimuli, but did not show any noteworthy interaction with cognitive reappraisal on any of our outcomes, contrary to the main hypothesis. These results indicate that $25 \mathrm{mg}$ oxazepam does not have a major effect on cognitive reappraisal. However, the effect on emotional stimuli (without regulation) indicates that oxazepam modulates the affective experience of negative stimuli. This finding is consistent with an earlier finding from our group, where i.v. midazolam reduced perceived unpleasantness of negative-valence IAPS images [48]. It has been suggested that this effect could be caused by a general decrease in anxiety due to inhibited amygdala-dependent emotional processing [12,25, 26]. Although the same physiological measurements were not used, our results contrast somewhat with previous reports that $20 \mathrm{mg}$ oxazepam did not modulate affective ratings or event-related potentials in response to emotional stimuli [49], and that neither 15 nor 30 mg oxazepam affected the fear-potentiated startle response [50]. Our results are however consistent with a finding that 0.25 and $1 \mathrm{mg}$ alprazolam inhibited startle responses to emotional stimuli [51].

Cognitive reappraisal works through reappraising emotional stimuli in different ways. Functional imaging studies have shown that dorsolateral prefrontal and lateral orbitofrontal cortices are specifically involved in these processes [7,9 11. It has also been shown that these prefrontal processes interact with processing of emotional information in brain regions such as the amygdala 52 54]. Since we showed effects of cognitive reappraisal on affective ratings and physiological measures but no interaction with benzodiazepines our data suggests that these regulatory processes do not interact but work in parallel. Therefore, our data do not corroborate the prediction that top-down regulatory mechanisms are suppressed by benzodiazepines, arising from the disinhibition theory of criminal violent behavior as suggested by [4,5]. The findings also do not suggest that benzodiazepines, although they have other risks in a clinical setting, contribute to poorer cognitive reappraisal in patients, as might have been expected from the observation that areas in the prefrontal cortex that are involved in emotional regulation also have high concentrations of $\mathrm{GABA}_{\mathrm{A}}$ receptors 28 .

Associations between on the one hand self-rated empathy, anxiety, alexithymia, psychopathy, and on the other hand cognitive reappraisal, have not been widely investigated. Exploratory analyses in our data showed that self-rated trait empathy measures were associated with stronger responses to negative-valence stimuli (except for personal distress that had the opposite result), whereas self-rated psychopathic traits were associated with weaker responses to negative-valence stimuli. Less consistent effects were observed in relation to instructions. One study has investigated the association of 
alexithymia to event-related potentials (ERP:s) during cognitive reappraisal, and found that higher alexithymia in a sample of healthy humans was associated with smaller ERP:s [55]. The strongest associations between rating scales and experimental outcomes were observed for self-rated unpleasantness. This may be explained by the similar nature between these self-rated measures, as opposed to physiological measures.

The generalizability of our results is limited by the nature of the sample, consisting of only male participants, most with ongoing or completed university education. This sample is not likely to be representative of benzodiazepine-prescribed patient groups nor recreational users. A further limitation concerns nature of the stimuli, which are a subset of all possible stimuli which could be used to induce emotion. The limited size of the sample, particularly the placebo group, precludes strong conclusions, and as with all randomised experiments, the possibility cannot be ruled out that baseline imbalances may influence the result, though we had no strong a priori reason to stratify randomisation to avoid imbalance on some particular variable. Furthermore, the choice of time windows for analyses of physiological signals is based on the observed data, which may introduce bias. Further work should use independent samples to define time windows of interest and to test hypotheses. It is also an open question whether different results would be seen with a higher dose of oxazepam or with another benzodiazepine.

\section{Conclusion}

While $25 \mathrm{mg}$ oxazepam caused lower rated unpleasantness in response to negative valence images, we did not observe an effect of $25 \mathrm{mg}$ oxazepam on cognitive reappraisal.

\section{Author contributions}

Conceived of study: GN, ST, AG, MI, PP. Collected data: GN, ST. Analysed data: GN. Interpreted results: GN, ST, AG, AO, KS, KH, MK, MI, PP. Drafted manuscript: GN. All authors read and approved the final version of the manuscript.

\section{Competing interests}

The authors declare that they have no competing interests.

\section{Acknowledgements}

We are grateful to Jonathan Berrebi for expert technical assistance. This work was supported by the Swedish Society for Medicine and the Osher Center for Integrative Medicine.

\section{References}

1. Etkin A, Büchel C, Gross JJ. The neural bases of emotion regulation. Nature Reviews Neuroscience. 2015 Nov;16(11):693-700.

2. Petrovic P, Castellanos FX. Top-Down Dysregulation-From ADHD to Emotional Instability. Frontiers in Behavioral Neuroscience. 2016;10:70.

3. Dåderman AM, Lidberg L. Flunitrazepam (Rohypnol) abuse in combination with alcohol causes premeditated, grievous violence in male juvenile offenders. The Journal of the American Academy of Psychiatry and the Law. 1999;27(1):83-99. 
4. Dåderman AM, Fredriksson B, Kristiansson M, Nilsson LH, Lidberg L. Violent behavior, impulsive decision-making, and anterograde amnesia while intoxicated with flunitrazepam and alcohol or other drugs: a case study in forensic psychiatric patients. Journal of the American Academy of Psychiatry and the Law Online. 2002 Jun;30(2):238-251. Available from: http://www.jaapl.org/content/30/2/238.

5. Dåderman AM, Fredriksson B, Nilsson LH, Kristiansson M, Lidberg L. The abuse of a sleeping pill that contains the active substance flunitrazepam (FZ)-for example, Rohypnol-and the effects of FZ intoxication, such as enhanced violence and disturbed memory. Journal of the American Academy of Psychiatry and the Law Online. 2004 Dec;32(4):467-468. Available from: http://www.jaapl.org/content/32/4/467.

6. Braunstein LM, Gross JJ, Ochsner KN. Explicit and implicit emotion regulation: a multi-level framework. Social Cognitive and Affective Neuroscience. 2017 Oct;12(10):1545-1557. Available from: https://academic.oup.com/scan/article/12/10/1545/4158838

7. Golkar A, Lonsdorf TB, Olsson A, Lindstrom KM, Berrebi J, Fransson P, et al. Distinct Contributions of the Dorsolateral Prefrontal and Orbitofrontal Cortex during Emotion Regulation. PLoS ONE. 2012 Nov;7(11):e48107. Available from: http://dx.doi.org/10.1371/journal.pone.0048107.

8. Buhle JT, Silvers JA, Wager TD, Lopez R, Onyemekwu C, Kober H, et al. Cognitive Reappraisal of Emotion: A Meta-Analysis of Human Neuroimaging Studies. Cerebral Cortex (New York, NY). 2014 Nov;24(11):2981-2990. Available from: http://www.ncbi.nlm.nih.gov/pmc/articles/PMC4193464/.

9. Kanske P, Heissler J, Schönfelder S, Bongers A, Wessa M. How to regulate emotion? Neural networks for reappraisal and distraction. Cerebral Cortex (New York, NY: 1991). 2011 Jun;21(6):1379-1388.

10. Eippert F, Veit R, Weiskopf N, Erb M, Birbaumer N, Anders S. Regulation of emotional responses elicited by threat-related stimuli. Human Brain Mapping. 2007 May;28(5):409-423.

11. Wager TD, Davidson ML, Hughes BL, Lindquist MA, Ochsner KN. Neural mechanisms of emotion regulation: Evidence for two independent prefrontal-subcortical pathways. Neuron. 2008 Sep;59(6):1037-1050. Available from: http://www.ncbi.nlm.nih.gov/pmc/articles/PMC2742320/.

12. Paulus MP, Feinstein JS, Castillo G, Simmons AN, Stein MB. Dose-dependent decrease of activation in bilateral amygdala and insula by lorazepam during emotion processing. Archives of General Psychiatry. 2005 Mar;62(3):282-288.

13. Schulze L, Domes G, Krüger A, Berger C, Fleischer M, Prehn K, et al. Neuronal correlates of cognitive reappraisal in borderline patients with affective instability. Biological Psychiatry. 2011 Mar;69(6):564-573.

14. Manber Ball T, Ramsawh HJ, Campbell-Sills L, Paulus MP, Stein MB. Prefrontal Dysfunction during Emotion Regulation in Generalized Anxiety and Panic Disorder. Psychological medicine. 2013 Jul;43(7):1475-1486. Available from: http://www.ncbi.nlm.nih.gov/pmc/articles/PMC4308620/. 
15. Rabinak CA, MacNamara A, Kennedy AE, Angstadt M, Stein MB, Liberzon I, et al. Focal and aberrant prefrontal engagement during emotion regulation in veterans with posttraumatic stress disorder. Depression and anxiety. 2014 Oct;31(10):851-861. Available from: http://www.ncbi.nlm.nih.gov/pmc/articles/PMC4141895/

16. Bishop SJ. Neurocognitive mechanisms of anxiety: an integrative account. Trends in Cognitive Sciences. 2007 Jul;11(7):307-316.

17. Picó-Pérez M, Radua J, Steward T, Menchón JM, Soriano-Mas C. Emotion regulation in mood and anxiety disorders: A meta-analysis of fMRI cognitive reappraisal studies. Progress in Neuro-Psychopharmacology \& Biological Psychiatry. 2017 Jun;79(Pt B):96-104.

18. Bowins B. Cognitive regulatory control therapies. American Journal of Psychotherapy. 2013;67(3):215-236.

19. Nutt D. GABAA receptors: subtypes, regional distribution, and function. Journal of clinical sleep medicine: JCSM: official publication of the American Academy of Sleep Medicine. 2006 Apr;2(2):S7-11.

20. Hörtnagl H, Tasan RO, Wieselthaler A, Kirchmair E, Sieghart W, Sperk G. Patterns of mRNA and protein expression for 12 GABAA receptor subunits in the mouse brain. Neuroscience. 2013 Apr;236:345-372. Available from: http://www.sciencedirect.com/science/article/pii/S0306452213000249.

21. Rudolph U, Crestani F, Möhler H. GABAA receptor subtypes: dissecting their pharmacological functions. Trends in Pharmacological Sciences. 2001

Apr;22(4):188-194. Available from: http://www.sciencedirect.com/science/article/pii/S0165614700016461.

22. Odano I, Halldin C, Karlsson P, Varrone A, Airaksinen AJ, Krasikova RN, et al. $[18 \mathrm{~F}]$ Flumazenil binding to central benzodiazepine receptor studies by PET: Quantitative analysis and comparisons with [11C]flumazenil -. NeuroImage. 2009 Apr;45(3):891-902. Available from: http://www.sciencedirect.com/science/article/pii/S1053811908012445.

23. Pringle A, Warren M, Gottwald J, Cowen PJ, Harmer CJ. Cognitive mechanisms of diazepam administration: a healthy volunteer model of emotional processing. Psychopharmacology. 2016 Jun;233(12):2221-2228. Available from: https://doi.org/10.1007/s00213-016-4269-y

24. Garcez H, Fernandes C, Barbosa F, Pereira MR, Silveira C, Marques-Teixeira J, et al. Effects of benzodiazepines administration on identification of facial expressions of emotion: a meta-analysis. Psychopharmacology. 2020

Jan;237(1):1-9. Available from: https://doi.org/10.1007/s00213-019-05393-y.

25. Brown GG, Ostrowitzki S, Stein MB, von Kienlin M, Liu TT, Simmons A, et al. Temporal profile of brain response to alprazolam in patients with generalized anxiety disorder. Psychiatry Research: Neuroimaging. 2015 Sep;233(3):394-401. Available from:

http://www.sciencedirect.com/science/article/pii/S0925492715300196.

26. Kreuder AK, Scheele D, Schultz J, Hennig J, Marsh N, Dellert T, et al. Common and dissociable effects of oxytocin and lorazepam on the neurocircuitry of fear. 
Proceedings of the National Academy of Sciences. 2020 May;117(21):11781-11787. Publisher: National Academy of Sciences Section: Biological Sciences. Available from: https://www.pnas.org/content/117/21/11781

27. Nilsonne G, Tamm S, Golkar A, Sörman K, Howner K, Kristiansson M, et al. Effects of $25 \mathrm{mg}$ oxazepam on emotional mimicry and empathy for pain: a randomized controlled experiment. Open Science. 2017 Mar;4(3):160607. Available from: http://rsos.royalsocietypublishing.org/content/4/3/160607.

28. Horder J, Andersson M, Mendez MA, Singh N, Tangen [U+FFFD] Lundberg J, et al. GABAA receptor availability is not altered in adults with autism spectrum disorder or in mouse models. Science Translational Medicine. 2018 Oct;10(461). Publisher: American Association for the Advancement of Science Section: Research Article. Available from: 10/ghtf2q.

29. Lang PJ, Bradley MM, Cuthbert BN. International affective picture system (IAPS): Affective ratings of pictures and instruction manual. Technical report A-8. 2008;.

30. Fridlund AJ, Cacioppo JT. Guidelines for Human Electromyographic Research. Psychophysiology. 1986;23(5):567-589. Available from: http://onlinelibrary. wiley.com/doi/10.1111/j.1469-8986.1986.tb00676.x/abstract.

31. Davis M. A multidimensional approach to individual differences in empathy. JSAS Catalog of Selected Documents in Psychology. 1980;10(85).

32. Davis MH. Measuring individual differences in empathy: Evidence for a multidimensional approach. Journal of Personality and Social Psychology. 1983;44(1):113-126.

33. Cliffordson C. Parents' Judgments and Students' Self-Judgments of Empathy. European Journal of Psychological Assessment. 2001 Jan;17(1):36-47. Available from: http://econtent.hogrefe.com/doi/abs/10.1027//1015-5759.17.1.36.

34. Spielberger C, Gorsuch R, Lushene P, Vagg P. Manual for the State-Trait Anxiety Inventory. Consulting Psychologists Press, Inc., Palo Alto, CA; 1983.

35. Nilsonne G. Data-and-analysis-code-Oxazepam-and-emotion: Release for publication. Zenodo; 2016. Available from: https://zenodo.org/record/60385\#.WWLIbYgrJnI

36. Bagby RM, Parker JDA, Taylor GJ. The twenty-item Toronto Alexithymia scale-I. Item selection and cross-validation of the factor structure. Journal of Psychosomatic Research. 1994 Jan;38(1):23-32. Available from: http://www.jpsychores.com/article/0022-3999(94)90005-1/abstract.

37. Simonsson-Sarnecki M, Lundh LG, Törestad B, Bagby RM, Taylor G, Parker J. A Swedish Translation of the 20-item Toronto Alexithymia Scale: Cross-validation of the Factor Structure. Scandinavian Journal of Psychology. 2000 Mar;41(1):25-30. Available from: http: //onlinelibrary.wiley.com/doi/10.1111/1467-9450.00167/abstract

38. Lilienfeld SO, Andrews BP. Development and Preliminary Validation of a Self-Report Measure of Psychopathic Personality Traits in Noncriminal Population. Journal of Personality Assessment. 1996 Jun;66(3):488-524. Available from: http://dx.doi.org/10.1207/s15327752jpa6603_3. 
39. Benning SD, Patrick CJ, Hicks BM, Blonigen DM, Krueger RF. Factor structure of the psychopathic personality inventory: validity and implications for clinical assessment. Psychological Assessment. 2003 Sep;15(3):340-350.

40. Sörman K, Nilsonne G, Howner K, Tamm S, Caman S, Wang HX, et al. Reliability and Construct Validity of the Psychopathic Personality Inventory-Revised in a Swedish Non-Criminal Sample - A Multimethod Approach including Psychophysiological Correlates of Empathy for Pain. PLOS ONE. 2016 Jun;11(6):e0156570. Available from: http://journals.plos.org/ plosone/article?id=10.1371/journal . pone.0156570

41. R Core Team. R: A Language and Environment for Statistical Computing. Vienna, Austria: R Foundation for Statistical Computing; 2015. Available from: http://www.R-project.org/

42. Lang DT. RCurl: General network (HTTP/FTP/...) client interface for R; 2015. $\mathrm{R}$ package version 1.95-4.6. Available from: http://CRAN.R-project.org/package=RCurl

43. Ryan JA. quantmod: Quantitative Financial Modelling Framework; 2015. R package version 0.4-4. Available from:

http://CRAN.R-project.org/package=quantmod.

44. Pinheiro J, Bates D, DebRoy S, Sarkar D, R Core Team. nlme: Linear and Nonlinear Mixed Effects Models; 2015. R package version 3.1-120. Available from: http://CRAN.R-project.org/package=nlme

45. Fox J. Effect displays in R for generalised linear models. Journal of Statistical Software. 2003;8(15):1-27.

46. Neuwirth E. RColorBrewer: ColorBrewer Palettes; 2014. R package version 1.1-2. Available from: http://CRAN.R-project.org/package=RColorBrewer

47. Ray RD, McRae K, Ochsner KN, Gross JJ. Cognitive Reappraisal of Negative Affect: Converging Evidence From EMG and Self-Report. Emotion (Washington, DC). 2010 Aug;10(4):587-592. Available from: http://www.ncbi.nlm.nih.gov/pmc/articles/PMC4106258/

48. Petrovic P, Dietrich T, Fransson P, Andersson J, Carlsson K, Ingvar M. Placebo in emotional processing-induced expectations of anxiety relief activate a generalized modulatory network. Neuron. 2005 Jun;46(6):957-969.

49. Olofsson JK, Gospic K, Petrovic P, Ingvar M, Wiens S. Effects of oxazepam on affective perception, recognition, and event-related potentials. Psychopharmacology. 2011 May;215(2):301-309. Available from: https://link.springer.com/article/10.1007/s00213-010-2141-z.

50. Baas JMP, Grillon C, Böcker KBE, Brack AA, Morgan CA, Kenemans JL, et al. Benzodiazepines have no effect on fear-potentiated startle in humans. Psychopharmacology. 2002 May;161(3):233-247.

51. Acheson DT, Stein MB, Paulus MP, Ravindran L, Simmons AN, Lohr JB, et al. Effects of anxiolytic treatment on potentiated startle during aversive image anticipation. Human Psychopharmacology. 2012 Jul;27(4):419-427.

52. Schiller D, Delgado MR. Overlapping neural systems mediating extinction, reversal and regulation of fear. Trends in Cognitive Sciences. 2010 Jun;14(6):268-276. Available from: 10/bvqpcr 
53. Milad MR, Quirk GJ. Fear Extinction as a Model for Translational Neuroscience: Ten Years of Progress. Annual review of psychology. 2012;63:129-151. Available from: 10.1146/annurev.psych.121208.131631.

54. Levy I, Schiller D. Neural Computations of Threat. Trends in Cognitive Sciences. 2021 Feb;25(2):151-171. Available from: 10/ghrm5n.

55. Walker S, O'Connor DB, Schaefer A. Brain potentials to emotional pictures are modulated by alexithymia during emotion regulation. Cognitive, Affective, \& Behavioral Neuroscience. 2011 Dec;11(4):463-475. Available from: https://link-springer-com.proxy.kib.ki.se/article/10.3758/ s13415-011-0042-1. 